\title{
Решение задачи оптимизации портфеля ценных бумаг с помощью методов многокритериальной оптимизации
}

\begin{abstract}
Киселева А.Ю. ${ }^{8}$
В статье исследуются компоненты финансовой отчетности, включение в систему ограничений которых улучшает авторскую модель многокритериальной оптимизачии портфеля ценных бумаг. Критически анализируются две недавно появившиеся модели многокритериальной оптимизации портфеля ценных бумаг.

Основоположником современной теории портфельного инвестирования является Г. Маркович [Markowitz H., 1952]. Также в портфельную теорию внесли значительный вклад Шарп [Sharp W., 1963], Tобин [Tobin J., 1958, 1965], Мертон [Merton R.,1973], Pocc [Ross S., 1976]. Эта теория непрерывно совершенствуется.

C развитием математического аппарата и вычислительных возможностей появились новые варианты поиска решений по оптимизации портфелей.

В 70-80-х годах XX века бурное развитие получили методы многокритериальной оптимизачии. Этот аппарат применялся автором при исследовании задачи оптимизациии портфелей цеенных бумаг.
\end{abstract}

\section{Метод группового учета аргументов (МГУА)}

Основоположником и разработчиком МГУА является академик Ивахненко А.Г. [Ивахненко А. Г., 1975, с. 29]. Его метод МГУА основан на теории самоорганизации, которая улучшает метод регрессионного анализа и приспосабливает его для так называемого прямого моделирования сложных систем по небольшому числу экспериментальных данных. МГУА представляет собой объединение регрессионного анализа и способов регуляризации. [Ивахненко А. Г., 1975, с. 29]

Существенным, по Ивахненко, является принцип единственности математической модели, получаемой по МГУА, для каждого заданного множества входных сигналов. Кроме того, МГУА позволяет получить математические модели оптимальной сложности и точности. Ивахненко экспериментально проверил, что оптимальная сложность модели достигается при учете запаздывающих аргументов и при высокой степени полного полинома регрессии. МГУА позволяет решать задачи, имеющие до 1000 аргументов. В это число входят как переменные, измеряемые в

\footnotetext{
${ }^{8}$ МГУ им. М.В. Ломоносова, соискатель кафедры «Финансы и кредит».
} 
данный момент, так и измеренные со сдвигами во времени (для учета предыстории) [Ивахненко А. Г., 1975, с. 29-30] (здесь и далее относительно теоретического представления МГУА цитируем автора метода, Ивахненко).

Большинство интерполяционных задач, решаемых с помощью МГУА, сводится к прямому восстановлению функции по небольшому количеству заданных ее точек (узлов интерполяции).

В задачах прогнозирования и распознавания образов всякая «протекция» (исключение неэффективных переменных) снижает эффективность.

Алгоритмы МГУА воспроизводят схему массовой селекции. В них есть генераторы усложняющихся из ряда в ряд комбинаций и пороговые самоотборы лучших из них. Так называемое «полное» описание объекта $\varphi=f\left(x_{1}, \ldots, x_{m}\right)$, где $\mathrm{f}$ - некоторая элементарная функция.

Входные аргументы и промежуточные переменные сопрягаются попарно, и сложность комбинаций на каждом ряду обработки информации возрастает (как при массовой селекции), пока не будет получена модель оптимальной сложности.

Каждое частное описание является функцией только двух аргументов. Поэтому его коэффициенты легко определить по данным обучающей последовательности при малом числе узлов интерполяции. Исключая промежуточные переменные (если это удается), можно получить «аналог» полного описания.

Из ряда в ряд селекции пропускается только некоторое количество самых регулярных переменных (для повышения обусловленности матриц полезно пропускать в последующие ряды селекции переменные первого ряда, т.е. использовать ковариации $x_{i} y_{i}, x_{i} z_{i} \ldots$ и т.д).

Степень регулярности оценивается по величине среднеквадратической ошибки (средней для всех выбираемых в каждом поколении переменных или для одной самой точной переменной) на отдельной проверочной последовательности данных.

Ряды селекции наращиваются до тех пор, пока регулярность повышается. Как только достигнут минимум ошибки, селекцию останавливают.

\section{Использование автором алгоритмов метода группового учета аргументов при анализе влияния показателей годовой финансовой отчетности на доходность простых акций через полгода после сдачи отчетности}

В исследовании использовалась БД Amadeus. Данные скачивались до конца декабря 2006 года. Для исследования первоначально были отобраны компании, находящиеся в первой сотне по величине активов. Из 
исходной выборки выбраковывались компании, по которым в БД не было данных по среднемесячным ценам акций, и случаи, в которых имелись пропуски по выбранным для анализа переменным.

Под примером будем понимать данные по всем отобранным переменным за какой- либо год (в нашем исследовании рассматривались данные с 2000-го по 2005 год, ). Вторичная выбраковка примеров проводилась по тем из них, в которых имелись пропуски по выбранным для анализа переменным. Для рассмотрения остался 181 пример.

Далее данные тестировались в NeuroShell 2. Тестирование показало низкий коэффициент $R^{2}$ при анализе взаимосвязи между зависимой (по нашей гипотезе) переменной «доходность акции» и остальными переменными как на тренировочном, так и на тестовом наборе при многократных экспериментах по подборке параметров нашей полиномиальной сети. Автор выдвинула рабочую гипотезу о необходимости примеров с «негативными» для данного показателя данными (нулевые или отрицательные переменные типа $x_{2}, x_{3}, x_{4}, x_{13}$ ). Таковых примеров оказалось 56. Переменная Gross Margin была убрана из рассмотрения ввиду большого числа пропусков данных по ней.

Поскольку нашей целью было выявление влияющих переменных из финансовой отчетности компаний, то, естественно, результаты на экспериментальном наборе несколько хуже, чем могли бы быть при включении в модель показателей курса акций компании (на май года, следующего за датой отчетного года). Для подавляющего большинства компаний май — это период оглашения финансовых результатов за отчетный год. Но показатель курсовой стоимости «перетягивает »прогнозную модель на себя, т.е. остальные переменные из уравнения вытесняются, и исследовать влияющие на доходность факторы из финансовой отчетности не представляется возможным.

\section{Справка из контекстуальной помощи NeuroShell 2}

\section{PSE (Prediction Squared Error - Квадратичная ошибка} предсказания)

Чтобы избежать разделения данных на два набора, с одной стороны, и чтобы избежать переучивания модели, с другой стороны, этот критерий представляет собой сумму двух членов: Норм. СКО (нормированный средний квадрат ошибки) и штраф за переучивание. (В литературе Норм. СКО часто также называют TSE - Training Squared Error, квадратичная ошибка тренировки.) Норм. СКО представляет собой средний квадрат ошибки модели на тренировочном наборе. Значение Норм. СКО отображается в модуле «Обучение» в текстовом поле «Норм. СКО» рамки «Статистика». Если вы хотите знать, чему равен штраф за переучивание, можете вычислить его как разность между Наилучшим 
значением критерия и Норм. СКО (это справедливо также для критериев FCPSE и MDL).

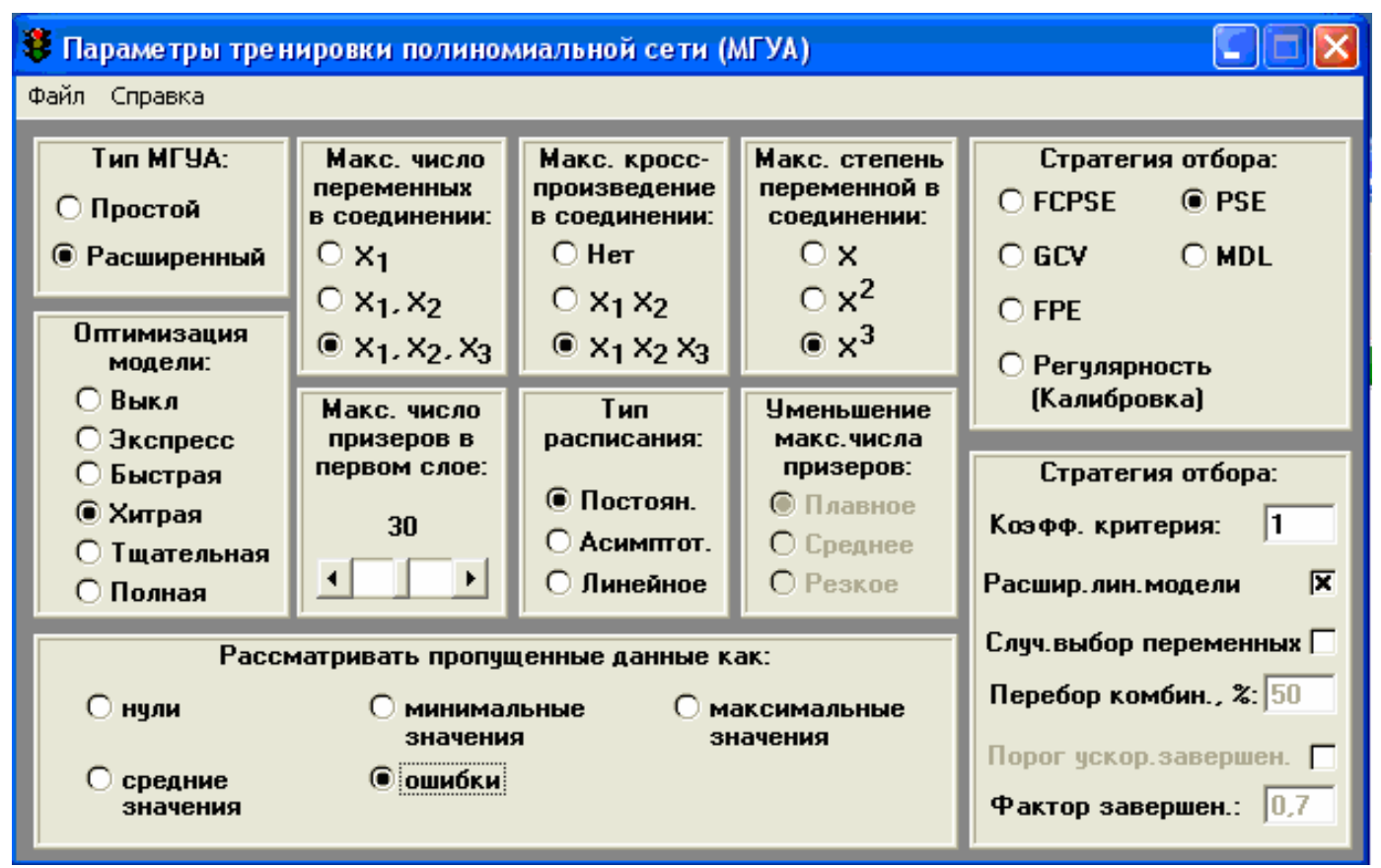

Рис.1. Параметры сети

Пусть N — количество примеров в файле данных, a k — количество коэффициентов в модели (определяемых таким образом, чтобы минимизировать Норм. СКО). Тогда PSE может быть записан как

$$
P S E=\text { Норм.СКО }+2 * \operatorname{var}(p) * \frac{k}{N},
$$

где $\operatorname{var}(\mathrm{p})$ - априорная оценка истинной дисперсии ошибки предсказывающей модели. Теоретическое рассмотрение показывает, что хорошей оценкой является $\operatorname{var}(\mathrm{p})=\operatorname{var}(\mathrm{a}) / 2$ (здесь $\operatorname{var}(\mathrm{a})-$ дисперсия реальной выходной переменной). Тем не менее имеет смысл ввести специальный множитель под названием КК (Коэффициент критерия), чтобы иметь возможность изменять вес штрафа за переучивание. Таким образом, окончательное выражение для PSE принимает вид

$$
P S E=\text { Норм.СКO+KK } * \operatorname{var}(a) * \frac{k}{N} .
$$




\section{Приводим данные из отчета полиномиальной сети}

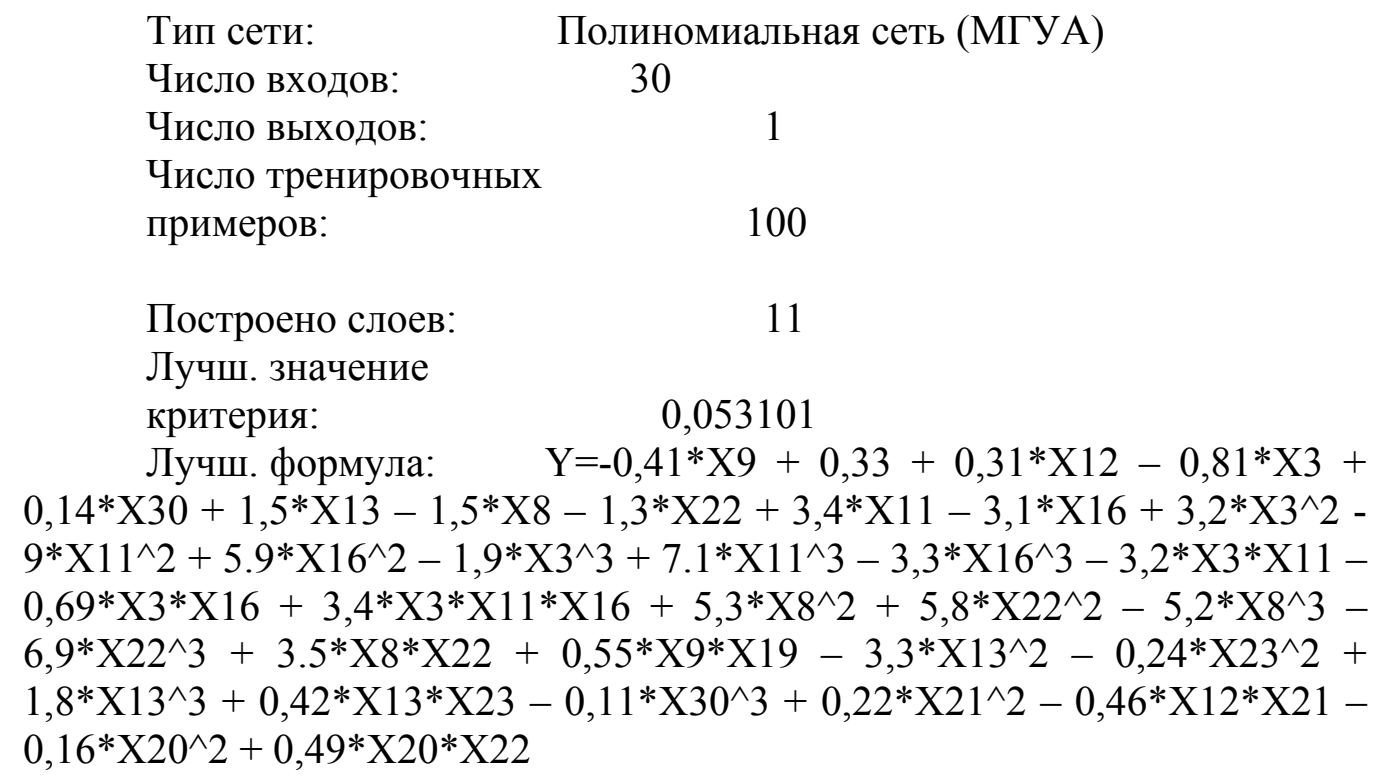

Обозначения:

$\mathrm{X} 1=$ (Operating revenue $/$ turnover $)$

$\mathrm{X} 2=($ Profit (loss) before tax $)$

$\mathrm{X} 3=(\mathrm{P} / \mathrm{L}$ for Period $[=$ Net Income $])$

$\mathrm{X} 4=($ Cash flow $)$

$\mathrm{X} 5=($ Total assets $)$

$\mathrm{X} 6=($ Shareholders funds $)$

$\mathrm{X} 7=($ Current ratio $)$

$\mathrm{X} 8=($ Liquidity ratio $(\%))$

$\mathrm{X} 9=($ Shareholders liquidity ratio $(\%))$

$\mathrm{X} 10=($ Solvency ratio $(\%))$

$\mathrm{X} 11=($ Gearing $(\%))$

$\mathrm{X} 12=($ Share funds per employee (Ths.) $)$

$\mathrm{X} 13=$ Work. capital per employee(Ths.)

$\mathrm{X} 14=($ Total assets per employee (Ths.)

$\mathrm{X} 15=($ Profit margin $(\%))$

$\mathrm{X} 16=($ Return on shareholders funds $(\%))$

$\mathrm{X} 17=($ Return on capital employed $(\%))$

$\mathrm{X} 18=($ Return on total assets $(\%))$

$\mathrm{X} 19=($ Interest cover $)$

$\mathrm{X} 20=($ Stock turnover $)$

$\mathrm{X} 21=($ Collection period (days) $)$

$\mathrm{X} 22=($ Credit period (days) $)$

$\mathrm{X} 23=($ Net assets turnover $)$

$\mathrm{X} 24=($ Costs of employees/oper. rev. $(\%))$ 
$\mathrm{X} 25=($ Operat. rev. per employee (Ths.))

$\mathrm{X} 26=$ Aver. cost of empl./year (Ths.))

$\mathrm{X} 27=$ Profit per employee (Ths.))

$\mathrm{X} 28=($ Cash Flow/Turnover $(\%))$

X29 $=($ EBIT Margin $(\%))$

$\mathrm{X} 30=($ EBITDA Margin $(\%))$

$\mathrm{Y}=($ yeld $)$

Самые существ.переменные: P/L for Period

Shareholders liquidity ratio (\%)

Liquidity ratio (\%)

Gearing (\%)*

Share funds per employee (Ths.)

Work. capital per employee (Ths.)

Return on shareholders funds (\%)

Interest cover

Stock turnover

Collection period (days)

Credit period (days)

Net assets turnover

EBITDA Margin (\%)

Менее существ. переменные:Return on total assets (\%)

Solvency ratio (\%)

Return on capital employed (\%)

Profit margin (\%)

Current ratio

Operat. rev. per employee (Ths.)

Costs of employees/oper. rev. (\%)

Aver. cost of empl./year (Ths.)

Profit per employee (Ths.)

EBIT Margin (\%) 


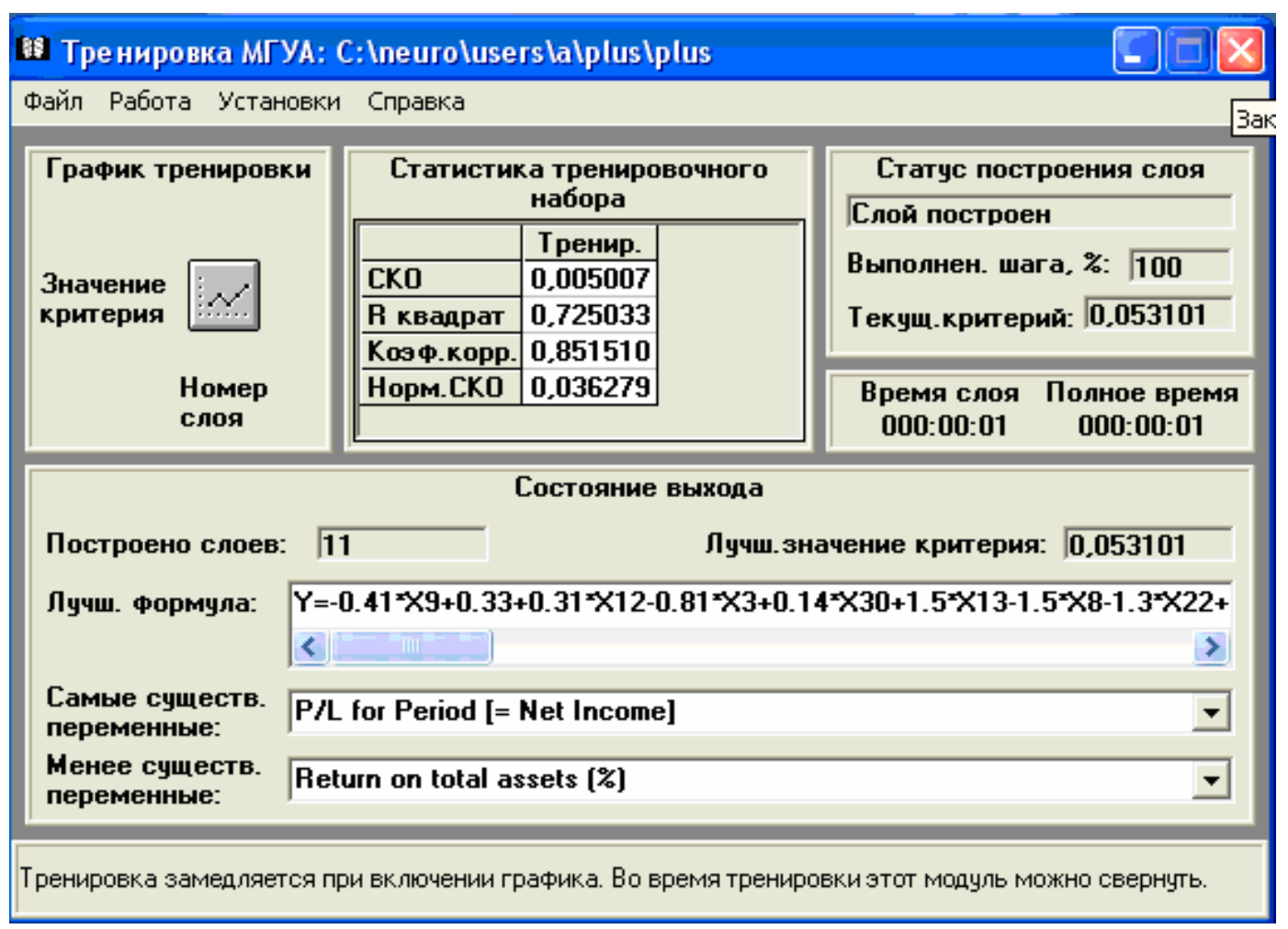

Рис.2. Снимок экрана по завершении тестирования на тренировочном наборе 


\section{Применение сети: $C$ :Ineuroluserslalplusiplus.pro Файл Работа Справка \\ Х Вычислять В квадрат и Т.А. (Выходы должны быть в файле данных) Ф Включать выходы в .ОUT файл [выходы должны быть в файле данных] Х Включать в .DUT файл разности между выходами и ответами сети}

Имя файла данных: C:Ineurołusersłałplus \plus.pro

Обработано примеров: 18

\begin{tabular}{||l|r|r|}
\hline Выход: & \multicolumn{1}{|c|}{ C1 } & \multirow{1}{*}{} \\
\hline R квадрат: & 0,5516 \\
\hline г квадрат: & 0,6029 \\
\hline Средний квадрат ошибки: & 0,0064 \\
\hline Ср. квадратичная ошибка [СК0]: & 0,080 \\
\hline Относительная СК0, \%: & 5,027 & \\
\hline Средняя абсолютная ошибка: & 0,0660 & \\
\hline Мин. абсолютная ошибка: & 0,0088 & \\
\hline
\end{tabular}

Применение сети к файлу данных (.pat) для получения выходного файла (.out).

Рис.3. Снимок экрана по завершении тестирования на экзаменационном наборе

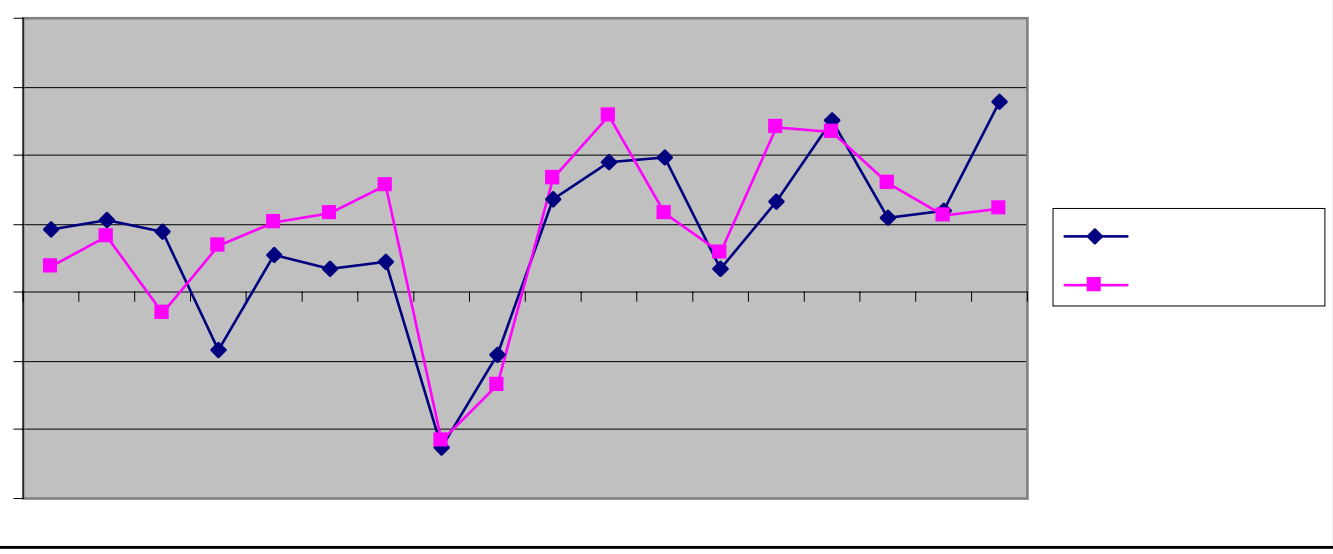

Рис.4. Прогнозирование полиномиальной сетью доходности по обыкновенным акциям отобранных компаний и фактические данные на экзаменационном наборе

В следующей статье мы продолжим рассказ об анализе примеров с негативными показателями (в расширенном списке компаний), так как применять нейронные сети на малых количествах примеров (менее 100) 
некорректно.

\section{Критический взгляд на новые постановки задач многокритериальной оптимизации портфеля ценных бумаг}

Остановимся подробнее на существенных результатах, полученных при решении задачи многокритериальной оптимизации портфеля ценных бумаг, полученных Койбаевой М. Х. Она решала задачу минимизации риска портфеля в трехмерном расширенном пространстве оптимальной кластеризации [Койбаева М. Х., 2006, с. 107] .

Минимизация риска портфеля начинается $\mathrm{y}$ Койбаевой $\mathrm{c}$ декомпозиции общей цели $\left(\min \sigma_{p}^{2}\right)$ Дисперсию портфеля $\sigma_{p}^{2}=\sum_{i=1}^{N} \sum_{j=1}^{N} w_{i} w_{j} \rho_{i j} \quad$ (где $\rho_{i j}$ - это ковариация между активами і и j) она использует в следующем, преобразованном виде:

(1)

$$
\sigma_{h}^{2}=\sum_{i=1}^{N} w_{i}^{2} \sigma_{i}^{2}+2 \sum_{i=1}^{N} \sum_{j>i}^{N} w_{i} w_{j} \sigma_{i} \sigma_{j} \rho_{i j}
$$

Койбаева в (1) производит следующую замену: $w_{i} \sigma_{i}=A_{i}$, $w_{j} \sigma_{j}=A_{j}$, и далее минимизирует целевую функцию (2):

$$
\sigma_{p}^{2}=\sum_{i=1}^{N} A_{i}+2 \sum_{i=1}^{N} \sum_{j>i}^{N} A_{i} A_{j} \rho_{i j}
$$

Но осях Х и Y находятся соответственно $A_{i}(\mathrm{i}=1, \ldots, \mathrm{N})$ и $A_{j}$ $(\mathrm{j}=1, \ldots, \mathrm{N}) . \quad$ А вот на оси $\mathrm{Z}$ находятся $\rho_{i j} \quad(\mathrm{i}=1, \ldots, \mathrm{N}, \mathrm{j}=1, \ldots, \mathrm{N})$. Упорядочивание пар чисел по оси $\mathrm{Z}$ в лексикографическом порядке, на наш взгляд, не является наилучшим способом организации данных, так как ведет к снижению качества модели - возможны другие варианты решений при перенумерации данных;целевые функции выглядят следующим образом [Койбаева М. Х., 2006, с.89]:

$$
\begin{aligned}
& A_{i} \rightarrow \min \\
& A_{j} \rightarrow \max \\
& \rho_{i j} \rightarrow-1
\end{aligned}
$$

Основная гипотеза Койбаевой, на которой базируется ее работа и которую возможно использовать при минимизации риска портфеля ценных бумаг, заключается в том, что для минимизации риска портфеля в кластеры объединяются не индивидуумы с близкими статистическими свойствами (как при кластерном анализе), а пары активов с наиболее 
далеко отстоящими статистическими свойствами [Койбаева М. Х., 2006, c.10].

В попытке решить новым способом задачу «увеличения среднеожидаемой доходности вложения при возможно меньшем риске» Шапкин А.С. и Шапкин В.А. обращаются к построению двух портфелей Марковица - Тобина: портфеля минимального риска и портфеля максимальной эффективности [Шапкин А.С. и Шапкин В.А., 2007, с.225].

Авторы монографии так ставят двухкритериальную задачу оптимизации портфеля инвестиций [Шапкин А.С. и Шапкин В.А., 2007, c.221]:

$$
\begin{gathered}
m(x)=x_{0} r_{0}+\sum_{i=1}^{N} x_{i} m_{i} \rightarrow \max \\
d(x)=\sum_{i, j=1}^{N} c_{i j} x_{i} x_{j} \rightarrow \min \\
\left\{\begin{array}{l}
\sum_{i=1}^{N} x_{i}=1 \\
x_{i} \geq 0 \\
i=0 \ldots N
\end{array}\right.
\end{gathered}
$$

(1)

Затем они обосновывают переход к следующей задаче, решение которой, по их мнению, является Парето-оптимальным для исходной двухкритериальной задачи (1) [Шапкин А.С. и Шапкин В.А., 2007, с.224]:

$$
\begin{array}{r}
\quad \sum_{i, j=1}^{N} c_{i j} x_{i} x_{j}-\lambda\left(x_{0} r_{o}+\sum_{i=1}^{N} x_{i} m_{i}\right) \rightarrow \min \\
\left\{\begin{array}{l}
\sum_{i=1}^{N} x_{i}=1 \\
x_{i} \geq 0 \\
i=0 \ldots N
\end{array}\right.
\end{array}
$$

(2)

Получилась так называемая задача с комбинированным функционалом.

Сначала рассмотрим, как трактуется коэффициент Лагранжа $\lambda$ на стр. 226 вышеупомянутой работы. По мнению авторов, это «весовой коэффициент, который численно выражает наше субъективное отношение 
к соотношению между доходностью и риском выполняемой операции. Если $\lambda=0$,то для нас важно осуществлять вложения с минимально возможным риском, даже если при этом доходность окажется минимальной. «Затем упоминается, что при выборе параметра $\lambda>1$ максимизируется значение средней доходности. Таким образом, авторы, очевидно, трактуют $\lambda$ как меру склонности инвестора к риску, которую можно выбрать априори.

С этим согласиться никак нельзя по следующим причинам. Вопервых, в отличие от склонности инвестора к риску, которая известна до составления портфеля, $\lambda$,будучи множителем Лагранжа, может быть вычислена.

Приведем необходимые пояснения к методу множителей Лагранжа по одной из базовых книг по математическому анализу для математиков - учебнику Зорича В.А. [Зорич, 2001, с.613].

При отыскании экстремума вещественнозначной функции $y=f\left(x^{1}, \ldots, x^{n}\right)$

от $\mathrm{n}$ переменных при условии, что эти переменные удовлетворяют системе уравнений

$$
\left\{\begin{array}{l}
F^{1}\left(x^{1}, \ldots \ldots x^{n}\right)=0, \\
\ldots \ldots \ldots \ldots \ldots . . . \\
F^{m}\left(x^{1}, \ldots \ldots x^{n}\right)=0
\end{array}\right.
$$

Можно написать с неопределенными множителями функцию Лагранжа:

$$
L(x, \lambda)=f(x)-\sum_{i=1}^{m} \lambda_{i} F^{i}(x)
$$

(3)

от $\mathrm{n}+\mathrm{m}$ переменных $(x, \lambda)=\left(x^{1}, \ldots x^{n}, \lambda_{1}, \ldots . \lambda_{m}\right)$. Далее можно вычислить уже критические точки функции (3). Для этого решается

$$
x_{0}=\left(x_{0}^{1}, \ldots, x_{0}^{n}\right)
$$

система (4) и ищутся

$$
\begin{aligned}
& \left\{\begin{array}{l}
\frac{\partial L}{\partial x^{j}}(x, \lambda)=\frac{\partial f}{\partial x^{j}}(x)-\sum_{i=1}^{m} \lambda_{i} \frac{\partial F^{i}}{\partial x^{j}}(x)=0 \\
\frac{\partial L}{\partial \lambda_{i}}(x, \lambda)=-F^{i}(x)=0 \\
(j=1, \ldots, n)
\end{array}\right. \\
& (i=1, \ldots, m)
\end{aligned}
$$

Как видно, речь не идет о подборе $\lambda$ произвольным образом (в 
зависимости от склонности инвестора к риску).

Теперь перейдем к неверной постановке задачи (2).

Разберемся с целевой функцией задачи (2). Она состоит из разности двух величин - уменьшаемого, представленного дисперсией искомого портфеля, и вычитаемого, представляющего собой ожидаемую доходность портфеля, скорректированную на безразмерную величину. То есть целевая функция лишена всякого экономического смысла. Оптимизировать в данном случае надо было целевую функцию от безразмерных величин, но господа Шапкины это проигнорировали, и получилось, что они решали некорректно поставленную задачу, к оптимизации портфеля в сформулированной ими постановке задачи отношения не имеющую.

Рассмотрим, какие рекомендации по этому вопросу дает Зайченко Ю.П. [Зайченко, 2004].

Поскольку целевые функции имеют различную размерность, то необходимо ввести некоторое преобразование $w_{i}\left(f_{i}(x)\right)$, приводящее $f_{i}(x)$ к безразмерному виду. Это преобразование должно удовлетворять, по крайней мере, следующим требованиям:

a) учитывать необходимость минимизации отклонений от оптимальных значений по каждой целевой функции;

б) иметь общее начало отсчета и один порядок изменения значений на всем множестве допустимых альтернатив;

в) сохранять отношение предпочтения на множестве альтернатив, сравниваемых по совокупности целевых функций $f_{i}$, и тем самым не изменять множества эффективных альтернатив.

Последнее требование означает, что преобразование $w_{i}\left(f_{i}(x)\right)$ должно быть монотонным. В качестве такого преобразования можно выбрать одну из монотонных функций следующего вида (здесь используются следующие обозначения: $f_{i_{-} \min }, f_{i_{-} \max }$ - соответственно наименьшие значения максимизируемых и наибольшие значения минимизируемых целевых функций, достигаемые ими на множестве допустимых альтернатив).

a) $w_{i}\left(f_{i}(x)\right)=\left\{\begin{array}{l}\frac{f_{i}^{0}-f_{i}(x)}{f_{i}^{0}-f_{i_{-} \min }} \partial л я \forall i \in I_{1} \\ \frac{f_{i}(x)-f_{i}^{o}}{f_{i_{-} \max }-f_{i}^{0}} \partial л я \forall i \in I_{2}\end{array}\right.$ 
б) $w_{i}\left(f_{i}(x)\right)=\left\{\begin{array}{l}\frac{f_{i}^{0}-f_{i}(x)}{f_{i}^{0}} \text { для } \forall i \in I_{1} \\ \frac{f_{i}(x)-f_{i}^{o}}{f_{i}^{0}} \text { для } \forall i \in I_{2}\end{array}\right.$

в) $w_{i}\left(f_{i}(x)\right)=\overline{w_{i}^{\mu}}\left(f_{i}(x)\right)$

Предлагаемая автором постановка задачи многокритериальной оптимизации (расчеты по ней будут представлены в следующем номере).

$$
\begin{aligned}
& \sum_{i=1}^{N} \mu_{i} \vartheta_{i} \rightarrow \max \\
& V a R_{p} \rightarrow \min \\
& \left\{\begin{array}{l}
\varphi\left(\xi_{i}\right) \geq \xi_{i}^{\text {критич }}+n p{ }_{-} \text {bool }=1 \\
\psi\left(\eta_{i}\right) \geq \eta_{i}^{\text {критич }}-n p u \_b o o l=0 \\
\sum_{i=1}^{N} \vartheta_{i}=1 \\
\vartheta_{i}>0 \\
i=1 . . N
\end{array}\right.
\end{aligned}
$$

Обозначения:

$\vartheta_{i}$ — вес і-го актива в портфеле;

Bool - переменная, определяющая принадлежность к подгруппе «хороших» акций (только положительные значения по переменным типа $\left.x_{2}, x_{3}, x_{4,} x_{13} ; \mathrm{Bool}=1\right)$ или «подозрительных» $(\mathrm{Bool}=0)$ акций;

$\xi_{1}$ — показатели из группы отобранных по МГУА как самые существенные для подгруппы «хороших» акций;

$\eta_{i}$ - показатели из группы отобранных по МГУА как самые существенные для подгруппы «подозрительных» акций.

\section{Список литературы}

1. Ивахненко А.Г. Долгосрочное прогнозирование и управление сложными системами,1975. - Киев: «Техніка».

2. Койбаева М. Х. Кластерные методы минимизации риска портфеля ценных бумаг. Дисс. на соискание звания к.э.н. по специальности 08.00.13, 2006 г. 
3. Шапкин А.С., Шапкин В.А. Управление портфелем инвестиций ценных бумаг. - М.: Издательско-книготорговая компания «Дашков и К ${ }^{\circ} \gg, 2007$ г.

4. Зорич В.А. Математический анализ, ч.1. — М: МЦНМО, 2001 г.

5. Markowitz H. Portfolio selection// Journal of Finance. 1952, vol.7, № 1.

6. Sharp W. Simplified model for portfolio analysis// Management Sciences. 1963, vol.9, № 2.

7. Tobin J. Liquidity Preference as Behavior Towards Risk // Review of Economic Studies, 26, № 1(February 1958).

8. Tobin J. The Theory of Portfolio Selection. The Theory of Interest Rates// ed. By Hahn F. and Brechlin, London, Macmillan and Co., 1965.

9. Merton R. An International Capital Asset Pricing Model // Econometrica (September 1973).

10. Ross S. The Arbitrage Theory of Capital Asset Pricing // Journal of Economic Theory, 13, № 3 (December 1976).

11. http://iasa.org.ua/iso?lang=rus $\& \mathrm{ch}=1 \& \mathrm{sub}=4$ 